\title{
Therapeutic efficacy of a complex drug based on interferons for Actinobacillus pleuropneumonia in piglets
}

\author{
Sergey Shabunin, Aleksey Shakhov, Larisa Sashnina*, Yuliya Vladimirova, and Kristina \\ Kopytina \\ Federal State Budgetary Scientific Institution "All-Russian Veterinary Research Institute of \\ Pathology, Pharmacology and Therapy", 394087, Voronezh, Lomonosov 114b, Russian Federation
}

\begin{abstract}
The article presents the results of studying the therapeutic efficacy of the complex drug tsipropig based on recombinant porcine interferons for Actinobacillus pleuropneumonia of piglets at an industrial pig-breeding complex and its effect on nonspecific immunity. It has been found that the drug has pronounced therapeutic properties and an immunomodulatory effect due to the presence of the fluoroquinolone antibiotic ciprofloxacin in the composition of tsipropig, the immunocorrective activity of its constituent recombinant porcine interferons alpha and gamma and vitamins $\mathrm{E}$ and $\mathrm{A}$ possessing antioxidant properties.
\end{abstract}

\section{Introduction}

Actinobacillus pleuropneumonia (APP) is an infectious disease of pigs characterized by the development of hemorrhagic necrotizing pneumonia and serous fibrinous pleurisy caused by Actinobacillus pleuropneumoniae [1-3]. The main factors of its pathogenicity include capsular polysaccharides, lipopolysaccharides, transferrin-binding proteins of the outer membrane and Apx toxins (cytolysins): ApxI, ApxII, and ApxIII [4].

Actinobacillus pleuropneumonia causes significant economic damage resulting from the high morbidity and mortality of piglets, reduced performance and product quality, as well as the cost of prevention and control of infection $[2,3]$.

The onset of the disease is facilitated by the weakening of the body's resistance due to various stress factors: early weaning of piglets from sows, regrouping and transportation of animals, hypothermia or overheating of the body, veterinary and zootechnical measures [24].

The empirical use of antimicrobial agents for the treatment of bacterial infections has led to the emergence of multidrug-resistant microbes that reduces the efficacy of etiotropic therapy of infectious diseases $[5,6]$. The lack of effective control over infectious diseases using antibacterial drugs determines the relevance of searching for alternative approaches, in particular based on modulating the immune response, since the development and course

\footnotetext{
* Corresponding author: 1.yu.sashnina@mail.ru
} 
of many infectious and inflammatory processes is accompanied by impaired functioning of the body's immune system $[6,7]$.

Immunomodulatory therapy has a nonspecific protective effect against a wide range of pathogens, based not on direct suppression of microorganisms but on the activation of the immune system [5, 8]. Immunomodulators, selectively acting on the corresponding component of cellular or humoral nonspecific immunity, in varying degrees, change the activity of all parts of the immune system [9].

To reduce the formation and spread of multidrug resistance of microorganisms to antimicrobial drugs and to reduce the immunodepressive effect of antibiotics on the animal organism, it is urgent to design complex drugs with antibacterial and immunotropic activity $[10]$.

Currently, the most promising immunotropic drugs include interferon immunomodulators $[9,10]$ the use of which in combination with etiotropic drugs increases the efficacy of therapy for bacterial and viral infections [6, 7].

One of these drugs is tsipropig (manufactured by ProBioTech Research and Production Center LLC, Republic of Belarus) which contains an antibiotic of the fluoroquinolone group ciprofloxacin, a mixture of recombinant porcine interferons alpha and gamma, vitamins $\mathrm{A}$ and $\mathrm{E}$.

The objective of the research is to study the therapeutic efficacy of tsipropig in case of Actinobacillus pleuropneumonia in piglets at an industrial pig-breeding complex.

\section{Materials and methods}

The studies have been carried out on an industrial pig-breeding farm which is unfavorable in terms of Actinobacillus pleuropneumonia. For the experiment, there were selected 31 piglets at the age of 50-55 days with an acute course of the disease, manifested by depression, rapid and hoarse breathing, painful cough, discharge from the nasal openings, an increase in body temperature up to $40.5-41.0^{\circ} \mathrm{C}$ from which there were formed 2 analogous groups. The animals were kept in one section in the conditions generally accepted at the pig breeding complex.

Respiratory pathology in piglets was diagnosed in accordance with the Methodological Manual for the Diagnosis, Prevention and Treatment of Respiratory Diseases in Piglets [11].

The etiology of respiratory infections was detected on the basis of the results of bacteriological and molecular and biological (PCR) studies of pathological material.

The animals of the first experimental group $(n=16)$ were intramuscularly administered tsipropig at a dose of $0.1 \mathrm{ml} / \mathrm{kg}$, the animals of the second group $(\mathrm{n}=15)$ were administered enroflox $10 \%-0.5 \mathrm{ml} / 10 \mathrm{~kg}$. The drugs were intramuscularly administered once a day until clinical recovery. Blood was taken for immunological studies from 5 animals of each group before the administration of drugs and 15 days after their recovery.

Bacteriological studies were carried out by conventional methods [12], molecular and biological studies - by PCR using the appropriate approved test systems.

Blood absorption activity of neutrophils (PAN), phagocytic number (PhN) and phagocytic index $(\mathrm{PhI})$, metabolic activity of neutrophils were determined: stimulated (st) and spontaneous (sp) test with nitro blue tetrazolium (NBT), in serum - bactericidal (SBA), lysozyme (SLA) and complement (SCA) activity, total protein, protein fractions, the content of circulating immune complexes (CIC) in accordance with the Methodical Recommendations for the Assessment and Correction of the Immune Status of Animals [13], the coefficient of pathogenicity of the CIC according to [14 ], the content of total immunoglobulins (Ig) according to [15], the reserve index (RI) of phagocytic neutrophils and the coefficient of metabolic activity of neutrophils (CMAN) - according to [16]. 
Statistical processing of the obtained data was carried out using Statistica v6.1 program, the assessment of the reliability of differences according to the Student's t-test.

\section{Results and discussion}

During bacteriological examination of pathological material (lungs, bronchial lymph nodes), the causative agent of Actinobacillus pleuropneumoniae was isolated from piglets with pneumonia slaughtered for diagnostic purposes, and its genome was found in molecular and biological research.

The use of tsipropig provided recovery of $93.8 \%$ of piglets that occurred on day $3.0 \pm$ 0.22 . The average daily weight gain was $294.0 \pm 6.9 \mathrm{~g}$. When treating with enroflox $10 \%$, the therapeutic efficacy was $86.7 \%$ with an average daily weight gain of $287.0 \pm 5.7 \mathrm{~g}$. Recovery occurred on day $5.0 \pm 0.20$.

When studying the immune status of piglets of both groups before treatment, there were recorded no significant differences in its indicators. There was detected a low activity of nonspecific humoral immunity factors (bactericidal, lysozyme and complement activity), as well as a high content of CICs of different dispersion that was associated with intoxication of the body and impaired homeostasis caused by the hemolytic and cytotoxic effects of APP toxins $[1,4]$.

The use of drugs in piglets had a positive effect on nonspecific humoral and cellular immunity (Table).

Table. Indicators of nonspecific immunity in piglets before and after treatment.

\begin{tabular}{|c|c|c|c|}
\hline \multirow[b]{2}{*}{ Indicators } & \multirow[b]{2}{*}{ Baseline } & \multicolumn{2}{|c|}{ Groups of animals } \\
\hline & & $\begin{array}{l}\text { experimental } \\
\text { (tsipropig) }\end{array}$ & $\begin{array}{c}\text { control } \\
\text { (enroflox } 10 \%)\end{array}$ \\
\hline SBA, $\%$ & $72.3 \pm 2.31$ & $82.8 \pm 2.57^{* *}$ & $81.0 \pm 4.76$ \\
\hline SLA, $\mu \mathrm{g} / \mathrm{ml}$ & $1.42 \pm 0.09$ & $1.96 \pm 0.07^{* *}$ & $1.8 \pm 0.19^{*}$ \\
\hline SCA, \% hem. & $3.78 \pm 0.09$ & $6.76 \pm 0.55^{* * *}$ & $4.67 \pm 0.5$ \\
\hline Total Ig $\mathrm{g} / \mathrm{L}$ & $21.0 \pm 1.65$ & $22.5 \pm 1.19$ & $22.7 \pm 1.25$ \\
\hline $\mathrm{CIC}, 3.0 \% \mathrm{mg} / \mathrm{ml}$ & $0.32 \pm 0.02$ & $0.33 \pm 0.026$ & $0.33 \pm 0.03$ \\
\hline $\mathrm{CIC}, 3.5 \% \mathrm{mg} / \mathrm{ml}$ & $0.36 \pm 0.029$ & $0.25 \pm 0.02^{* *}$ & $0.30 \pm 0.015^{*}$ \\
\hline $\mathrm{CIC}, 4,0 \% \mathrm{mg} / \mathrm{ml}$ & $0.42 \pm 0.03$ & $0.30 \pm 0.018^{* *}$ & $0.33 \pm 0.028^{*}$ \\
\hline $\mathrm{C} 4 / \mathrm{C} 3$ & $1.3 \pm 0.02$ & $0.9 \pm 0.02^{* * *+}$ & $1.0 \pm 0.03$ \\
\hline PAN, $\%$ & $74.0 \pm 1.38$ & $75.2 \pm 1.09$ & $68.8 \pm 0.8^{* *}$ \\
\hline $\mathrm{PhI}$ & $5.2 \pm 0.22$ & $7.9 \pm 0.36^{* * *+++}$ & $5.1 \pm 0.19$ \\
\hline $\mathrm{PhN}$ & $3.8 \pm 0.23$ & $5.9 \pm 0.3^{* * *+++}$ & $3.6 \pm 0.17$ \\
\hline sp-NBT & $15.2 \pm 1.56$ & $19.4 \pm 0.97^{*++}$ & $15.4 \pm 0.87$ \\
\hline st-NBT & $24.6 \pm 1.02$ & $35.2 \pm 1.07^{*+}$ & $26.8 \pm 0.58$ \\
\hline RI & $1.6 \pm 0.07$ & $1.8 \pm 0.02^{*}$ & $1.74 \pm 0.04$ \\
\hline CMAN & $0.38 \pm 0.02$ & $0.45 \pm 0.026^{*}$ & $0.42 \pm 0.03$ \\
\hline
\end{tabular}

Note: ${ }^{*} \mathrm{P}<0.05 ;{ }^{* *} \mathrm{P}<0.001 ;{ }^{* * *} \mathrm{P}<0.0001$ relative to the baseline studies ${ }^{+} \mathrm{P}<0.05 ;{ }^{++} \mathrm{P}<0.001 ;{ }^{+++} \mathrm{P}$ $<0.0001$ - relative to the indicators of the control group

After treatment, the piglets of the experimental and control groups demonstrated an increase in comparison with the baseline serum bactericidal activity by $14.5 \%$ and $12.0 \%$, indicating the active mobilization of the body's defense mechanisms in response to infection, an increase in lysozyme activity - by $38.0 \%$ and $26.8 \%$ reflecting the high proliferative activity of lysozyme-synthesizing cells. In the piglets of the experimental group, SLA exceeded the control indicator by $8.9 \%$. 
After treatment with tsipropig, serum complement activity in piglets increased by $78.8 \%$ compared to the baseline and was by $44.8 \%$ higher than the control value that increased less significantly (by $23.5 \%$ ).

The content of total immunoglobulins in piglets of the experimental and control groups after treatment was higher than the baseline value by $7.1 \%$ and $8.1 \%$, respectively.

The level of circulating immune complexes $(3 \%)$, formed as a result of the antigenantibody reaction and involved in maintaining the homeostasis of the body, did not change in the experimental animals after therapy. The number of CICs $(3.5 \%)$ and $(4 \%)$ in the piglets of the experimental and control groups decreased by $30.6 \%$ and $16.7 \%, 28.6 \%$ and $21.4 \%$, respectively, while in the piglets after the administration of tsipropig their content was less by $13.9 \%$ and $10.0 \%$ than in case of the treatment with enroflox $10 \%$.

As a result of a decrease in the concentration of finely dispersed forms of immune complexes, a decrease in the pathogenicity index $(\mathrm{C} 4 / \mathrm{C} 3)$ in piglets after the use of tsipropig by $30.8 \%$ and enrofloxacin - by $30.0 \%$ was detected, indicating their pronounced elimination from the body and a decrease in antigenic load. In the piglets of the experimental group, the pathogenicity index was by $10.0 \%$ lower than in the control that reflected the efficacy of tsipropig action.

The number of active phagocytes in the piglets of the experimental group slightly increased compared to the baseline but was by $9.3 \%$ higher than the control value that decreased by $7.0 \%$ compared to the baseline. After treatment with tsipropig, there was a significant increase in the absorption function of phagocytes, $\mathrm{PhI}$ and $\mathrm{PhN}$ - by 53.2 and $53.8 \%$ and exceeded similar indicators in the piglets of the control group, in which $\mathrm{PhI}$ did not change, by 54.9 and $63.0 \%$, and $\mathrm{PhN}$ decreased by $5.3 \%$ compared to the baseline values.

The metabolic activity of neutrophils in piglets after treatment with tsipropig, increased in spontaneous and stimulated NBT by $27.6 \%$ and $11.4 \%$, and as a result of therapy with enroflox $10 \%$ - by 1.3 and $8.9 \%$, respectively, compared with the baseline values. The level of neutrophil activity in the piglets of the experimental group in sp- and st-NBT was higher by $26.0 \%$ and $10.7 \%$ than the control values.

The functional reserve of cells and the index of activation of neutrophils were higher in piglets after the application of tsipropig by 12.5 and $18.4 \%$, and enrofloxacin - by 8.7 and $7.1 \%$ compared with the baseline, while in the animals of the experimental group RI and CMAN significantly exceeded similar indicators in the control one by 3.4 and $10.5 \%$.

The high efficacy of the use of tsipropig in case of Actinobacillus pleuropneumonia is due to the action of ciprofloxacin, which like enrofloxacin, is a broad-spectrum antimicrobial drug from the group of fluoroquinolones with high antimicrobial activity against gram-negative and gram-positive microorganisms during rest and division. The mechanism of the antimicrobial action of ciprofloxacin and enrofloxacin is due to a violation of the DNA synthesis of a microbial cell and is mediated by inhibition of the enzymes topoisomerase IV and DNA gyrase (topoisomerase II) of the bacterial cell that are responsible for the synthesis of replication, transcription, repair and recombination of bacterial DNA [26]. During the formation of the spatial structure of a DNA molecule during its replication, DNA gyrase catalyzes negative supercoiling of DNA strands, and topoisomerase IV participates in the decatenation of covalently closed circular DNA molecules. Inhibition of these enzymes disrupts the growth and division of the bacterial cell and leads to its death.

A feature of ciprofloxacin is low toxicity, lack of immunosuppression and activity against microorganisms with acquired resistance [27].

The efficacy of the antimicrobial action of the drug is increased by the immunomodulatory effect on the body, caused by recombinant porcine IFN- $\alpha$ and IFN- $\gamma$ that by increasing the activity of neutrophils, monocytes/macrophages regulate the 
migration of phagocytes into the inflammatory focus, enhance the cytotoxic properties of macrophages, death and intracellular bacteria [28]. IFN- $\alpha$ exhibits immunomodulatory properties, increasing the activity of natural killers, T-helpers, phagocytes, the intensity of differentiation of B-lymphocytes [29], as well as the antibacterial activity of macrophages [30]. In addition, the bacteriostatic effect of IFN- $\alpha$ is due to the disturbance of bioenergetic processes in bacteria (due to depletion of tryptophan), and the mediated bactericidal effect is due to the generation of nitric oxide and reactive oxygen species in macrophages [30, 31]. IFN- $\gamma$, being the most important anti-inflammatory cytokine, has a pronounced immunomodulatory effect, activates the effector functions of macrophages, cytotoxic $\mathrm{T}$ lymphocytes, natural killer cells, prostaglandin and corticosteroid systems, contributing to the effective elimination of the infectious agent [29, 32].

The use of tsipropig has contributed to an increase in the humoral and cellular indicators of nonspecific resistance of animals. Serum bactericidal activity, which characterizes the general state of the factors of nonspecific resistance of the organism, is an integral indicator that ensures the homeostasis of the organism and the normal course of physiological processes. The level of SBA depends on the active mobilization ability of biochemical and humoral inhibitory systems in relation to any structure with signs of genetic foreignness [17].

The activity of lysozyme, a muramidase enzyme synthesized and secreted by neutrophils, monocytes and macrophages, makes it possible to assess the functional state of the phagocytic link of immunity $[18,19]$. It has been found that lysozyme is involved in the regulation of immune and metabolic processes and also plays an important role both in stimulating the pro-inflammatory response and in its systemic limitation that leads to a decrease in the inflammatory process. In addition, extracellular lysozyme destroys multimeric peptidoglycans to soluble fragments that activate NOD receptors in epithelial cells of the mucous membrane that leads to the secretion of chemotactic factors of neutrophils and macrophages and additionally induces pro-inflammatory pathways [18]

Serum complement activity, being the most important indicator of nonspecific resistance, provides lysis of sensitized infectious agents and mediates cellular and humoral interactions within the immune response, including chemotaxis, phagocytosis, cell adhesion and B-cell differentiation [20]. Complement activation is carried out according to the classical, lectin and alternative pathways during which the formation of three main types of effectors occurs: anaphylatoxins (C3a and C5a), opsonins (C3b, iC $3 b$ and $\mathrm{C} 3 \mathrm{~d}$ ) and a membrane attack complex (MAC, C5b-9) that directly lyses opsonized pathogens or damaged own cells which is of great importance in protecting against gram-negative bacteria $[21,22]$.

Circulating immune complexes have an important pathogenetic and prognostic value, while large-molecular CICs are insoluble, have limited pathogenicity and are rapidly phagocytosed, and small immune complexes have a high pathological potential, are not able to activate complement and, as a result of weak elimination, are in the bloodstream for a long time that leads to depletion of neutrophils and weakening of immunological reactions [23].

Studies [24] have established a pronounced degree of CICs elimination under the effect of interferon-producing drugs, indicating a high degree of their immunomodulatory activity.

Phagocytic activity of neutrophils is crucial for the body's nonspecific defense against various pathogens due to the production of oxygen radicals and proteolytic enzymes that is enhanced when bacteria are opsonized by plasma factors such as antibodies and complement activation products (C3b, iC $3 b)$ [25]. 
The revealed changes in the metabolic activity of neutrophils indicated an increase in cytotoxicity and intracellular activity of phagocytes and an adequate state of the cellular link of nonspecific resistance.

Vitamins $\mathrm{A}$ and $\mathrm{E}$ included in the drug have antioxidant properties, regulate the synthesis and breakdown of phospholipids in case of normal states and in case of any pathological states, including infectious and inflammatory diseases, participate in the functioning of the immune system. In addition, with the participation of vitamin E, cell membranes are restored during inflammatory processes, and this prevents the destruction of both endogenous and exogenous IFN by cellular proteases.

\section{Conclusion}

The conducted studies have established that the use of the complex drug tsipropig in piglets with APP is accompanied by a more significant therapeutic effect due to the presence of an antibiotic with a wide spectrum of antimicrobial action, recombinant porcine interferons alpha and gamma with immunomodulatory activity and vitamins $A$ and $E$ in its composition. The use of tsipropig is accompanied by an increase in serum bactericidal, lysozyme and complement activity, the absorption and metabolic activity of neutrophils and the elimination of CICs of different dispersion that has contributed to an increase in the therapeutic efficacy and a reduction of the recovery time.

\section{References}

1. P. Martelli, H. Segalis, Respiratory diseases of pigs, Spain (2019)

2. A.V. Potekhin, V.F. Kovalishin, Actinobacillus pleuropneumonia of pigs: diagnosis, prevention and control measures, FGBU "VNIIZZh", Veterinary medicine today 3(10), $18: 29$ (2014)

3. M. Gottschalk, American Association of Swine Veterinarians 381, 84 (2007)

4. K. Chiers, T.De Waele, F. Pasmans, R. Ducatelle, F. Haesebrouck, Vet. Res. 41, 65 (2010) DOI: 10.1051/vetres/2010037

5. V.A. Bulgakova, I.I. Balabolkin, A.S., Ignatova, Consilium Medicum 18 (11), 96-101 (2016)

6. A.V. Karaulov, O.V. Kalyuzhin, Therapeutic Archives, 85:11, 100:08 (2013)

7. Yu.N. Fedorov, V.I. Klyukina, M.N. Romanenko, O.A. Bogomolov, A.N. Denisenko, Strategy and principles of immunocorrection and immunomodulatory therapy, Bulletin of Novgorod State University 86, 84-87 (2015)

8. R.I. Sepiashvili, Importance for immunorehabilitation, Allergology and immunology 16 (1), 70-74 (2015)

9. S.V. Zaytseva, O.V. Zaytseva, Acute respiratory infections in children: etiopathogenetic possibilities of modern therapy, breast cancer, 22 (2), 1520-25 (2014)

10. V.A. Prokulevich, M.I. Potapovich, Bulletin of BSU, 2 (3), 51-54 (2011)

11. A.G. Shakhov, L.Yu. Sashnina, D.V. Fedosov et al., Methodological manual for the diagnosis, prevention and treatment of respiratory diseases in piglets, (Voronezh, 2010)

12. M.A. Sidorov, D.I. Skorodumov, V.B. Fedotov, Specifier of zoopathogenic microorganisms (Moscow, Kolos, 1995) 
13. A.G. Shakhov, Yu.N. Brigadirov, A.I. Anufriev et al., Methodical recommendations for the assessment and correction of animal resistance, 32 (Voronezh, 2005)

14. Yu.V. Skibo, N.Sh. Kurmaeva, V.N. Tsibulkina, I.G. Mustafin, Z.I. Abramova, Kazan Med. Jour. 94 (5), 744-48 (2013)

15. I.P. Kondrakhin, A.V. Arkhipov, V.I. Levchenko, G.A. Talanov, L.A. Frolov, V.E. Novikov, Methods of Veterinary Clinical Laboratory Diagnostics, (M., Kolos, 2004)

16. A.I. Karpishchenko, A.N. Alekseev, V.V. Alipov, GEOTAR-Media, 2, (2013)

17. A.P. Polkovnichenko, V.P. Bykov, D.V. Vorobiev [et al.], Natural Sciences, 1 (38), 160-63 (2012)

18. M.S. Zharkova, D.S. Orlov, O.Y. Golubeva, O.B. Chakchir, I.E. Eliseev, T.M. Grinchuk, O.V. Shamova, Front Cell Infect Microbiol, 30, 9-128 (2019) doi: 10.3389/fcimb.2019.00128.

19. S.A. Ragland, A.K. Criss, PLoS Pathog 13 (9), e1006512 (2017) https://doi.org/10.1371/ journal.ppat.1006512

20. Y.M. Ali, N.J. Lynch, K.S. Haleem, T. Fujita, Y. Endo, et al., PLoS Pathog 8 (7), e1002793 (2012) doi:10.1371/journal.ppat.1002793

21. L. Skattum, M. van Deuren, T. van der Poll, L. Truedsson, Molecular Immunology, 48 (14), 1643-55 (2011) doi.org/10.1016/j.molimm.2011.05.001

22. P. Cravedi, W. van der Touw, P.S. Heeger, Complement Regulation of T-Cell Alloimmunity 33 (6), 565-574 (2013) doi.org/10.1016/j.semnephrol.2013.08.007

23. I.I. Ivanov, E.N. Prochan, M.V. Cheripko, N.V. Kosolapova, Crimean Journal of Experimental and Clinical Medicine, 1 (3-4), 37-42 (2011)

24. S.A. Mashkova, L.V. Kolobukhina, T.P. Ospelnikova., L.N. Merkulova, F.I. Ershov, International journal on immunorehabilitation. Issue Physiology and Pathology of the Immune System, 6 (2-1), 66 (2004)

25. Y-P Ko, A Kuipers, CM Freitag, I Jongerius, E Medina, et al, PLoS Pathog 9(12), e1003816 (2013) doi:10.1371/journal.ppat.1003816

26. V.N. Tsarev, R.V. Ushakov, E.V. Ippolitov, S.E. Brodskiy, Dentistry for all. 4, 14-19 (2006)

27. N.G. Berdnikova, RMJ, 5, 351 (2007)

28. A.G. Shakhov, L.Yu. Sashnina, M. Yu. Zheynes, Yu.Yu. Vladimirova, N.V. Karmanova, Bulletin of Veterinary Pharmacology 2 (11), 49-60 (2020) DOI: 10.17238/issn2541-8203.2020.2.49

29. T.V. Sologub, V.V. Tsvetkov, E.G. Deeva, Rus. Medicobiological Bulletin named after Acad. I.P. Pavlov 3, 56-60 (2014)

30. GM Boxx, G. Cheng, Cell Host Microbe, 19(6), 760-69 (2016) doi:10.1016/j.chom.2016.05.016

31. S.V. Shabunin, A.G. Shakhov, G.A. Vostroilova, L.V. Cheskidova, P.A., Parshin T.I. Ermakova, N.A. Grigoryeva, Agricultural Biology, 53 (4), $851-59$ (2018) doi: 10.15389/agrobiology.2018.4.851eng

32. H.M. Johnson, Front. Immunol., 5, 667, 2015 doi: 10.3389/fimmu.2014.00667 